\title{
Diseño y producción de recursos didácticos digitales mediante Plotagón en el Grado de Educación Infantil
}

\section{Design and production of digital educational resources through Plotagon in the Degree of Early Childhood Education}

\author{
María Dolores Hidalgo Ariza ${ }^{1}$, Juan Manuel Muñoz González², Esther María Vega Gea ${ }^{3}$ \\ ${ }^{1}$ Departamento de Educación, Universidad de Córdoba, España (lola.hidalgo@uco.es) \\ ${ }^{2}$ Departamento de Educación, Universidad de Córdoba, España (juan.manuel@uco.es) \\ ${ }^{3}$ Departamento de Educación, Universidad de Córdoba, España (esther.vega@ uco.es)
}

Recibido el 30 de octubre de 2017; revisado el 30 de noviembre de 2017; aceptado el 25 de septiembre de 2018 ; publicado el 1 de junio de 2019

\section{RESUMEN:}

El alumnado del siglo XXI demanda nuevas metodologías de aprendizaje donde el uso de las tecnologías de la información y comunicación se plantea como un recurso ineludible dentro del aula. Esta cuestión conlleva a que tanto la formación inicial docente como la permanente contemplen, dentro de sus programas formativos, diferentes aspectos que permitan a los profesionales de la educación el desarrollo de las capacidades, habilidades y destrezas necesarias para la integración de dichos recursos en dinámicas de aprendizaje de cualquier etapa educativa (Escudero, Martínez-Domínguez y Nieto, 2018). En este sentido, presentamos un estudio, enmarcado dentro de la educación superior, concretamente en el Grado de Educación Infantil de la Universidad de Córdoba, donde el alumnado debe aprender a utilizar la aplicación Plotagón para diseñar y producir recursos didácticos audiovisuales, que contemplen los diferentes elementos curriculares de dicha etapa. El estudio lo abordamos desde una metodología cuantitativa, a través del uso de un cuestionario creado ad hoc y conformado por 27 ítems que versarán sobre el aprendizaje del software Plotagón en educación superior. Los resultados obtenidos en esta investigación muestran una postura de acuerdo del alumnado respecto a las percepciones y las aportaciones al aprendizaje de los recursos audiovisuales; sin embargo, su opinión en cuanto a la valoración del programa Plotagón utilizado para la elaboración de los mismos es de indiferencia.

PALABRAS CLAVE: EDUCACIÓN INFANTIL, RECURSOS DIDÁCTICOS, TIC, TECNOLOGÍA EDUCATIVA, FORMACIÓN INICIAL DOCENTE.
ABSTRACT:
In the XXI century, students demand new learning methodologies in which the use of Information and Communication Technologies into the classroom is considered as inescapable. This question entails that both initial teacher training and permanent training should consider including, inside their training programs, contents that enable teachers to develop their abilities and skills for integrating this kind of resources with learning dynamics in any educational stage. In this line, here we present a study, framed in the University Education, specifically in the Degree on Children Education from the University of Córdoba (Spain), where students have to learn to use the Plotagon computer application in order to design and produce audiovisual didactic resources that involve the different curricular elements in this stage. A quantitative approach has been used in this research, through a questionnaire created ad hoc that is composed by 27 items regarding the learning of the Plotagon software in High Education. The obtained results show the students agreement in 
terms of perception of the contributions of this audiovisual resources to the learning process. However, their opinion about the assessment of this application can be ranked as indifferent.

KEYWORDS: Children education, didactic resources, ICT, educational technology, initial teacher training.

\section{INTRODUCCIÓN}

El crecimiento tecnológico conlleva inevitablemente una serie de cambios en la sociedad puesto que, prácticamente, la totalidad de áreas de desarrollo del individuo se encuentran, de alguna manera, influenciadas por las tecnologías de la información y la comunicación (TIC) (Marín, 2014; Marín y Sampedro, 2016). Esta transformación social ha generado nuevas formas de relación, comunicación $\mathrm{y}$, en definitiva, una reciente forma de vida (Vásquez-Cuperio y López-Penedo, 2016) que se ha denominado sociedad del conocimiento. Esta ha supuesto una mejora continua en los procesos de formación y aprendizaje, todo ello impulsado por el uso de las TIC (Blanco y Anta, 2016). En este contexto se requieren profundos cambios en el ámbito educativo, en el que surjan nuevas metodologías y herramientas orientadas a potenciar los procesos de enseñanza y aprendizaje mediante la aplicación de las TIC (González, García y Gonzalo, 2011).

En el escenario descrito, la única manera de afrontar estas recientes exigencias es la transformación de los espacios académicos y materiales pedagógicos, con objeto de preparar tanto a estudiantes como a docentes mediante conocimientos, habilidades y destrezas demandadas en la sociedad digital (Adell, 2004). Este cambio presenta una especial relevancia en la educación superior, donde se resaltan (Maldonado, 2012) los nuevos roles de profesorado y alumnado, surgidos tras la inclusión de las TIC en la docencia universitaria. Mediante la interacción con estas tecnologías, se permiten distintas representaciones de la información, facilitando diversas formas de expresión y participación y dando cobertura a las múltiples facetas del aprendizaje y la enseñanza (Sancho y Hernández, 2006).

Sin embargo, para poder implementar estos nuevos procesos formativos y modelos pedagógicos, de gran potencial innovador, es indispensable dotar al profesorado con los conocimientos necesarios (Santiago, Navaridas y Reparaz, 2014) para aprovechar todos los recursos metodológicos y tecnológicos de manera que puedan incorporarlos en su práctica profesional (Cabero y Marín, 2012; Fernández y Barreira, 2017). Por ello, hay que resaltar la necesidad de incorporar estos saberes en los programas de formación inicial docente (Cabero, 2010), de forma que se prepare a los futuros maestros para el escenario de la educación digital.

Dentro del ámbito de los actuales modelos pedagógicos, cabe destacar el cambio en la educación centrada en la mera transmisión de conocimientos, en la que el estudiante tomaba un papel exclusivamente pasivo, hacia un nuevo enfoque metodológico de aprendizaje activo. En este tipo, los estudiantes toman un papel central dentro del proceso de adquisición de conocimiento y el profesorado ejerce las labores de guía y facilitador de herramientas y recursos a través de las TIC (Boza y Conde, 2015). Esto contribuye a una mayor implicación del alumnado y a la mejora de los resultados (Dans y Muñoz, 2016), por lo que muchos países europeos recomiendan este tipo de modelos, como son el aprendizaje por proyectos, la enseñanza individualizada o la investigación científica (EURYDICE, 2011). Por tanto, el docente debe estar familiarizado con estas tecnologías, saber qué recursos existen, dónde se pueden encontrar y saber integrarlos en su actividad diaria (Meter, 2004).

Por otro lado, estas herramientas presentan una importancia fundamental para el desarrollo de estos nuevos enfoques (Roblizo y Cózar, 2015). En general, la legislación en materia de planes de estudios para futuros profesores es rica en estrategias pedagógicas, pero puede presentar carencias con relación a las herramientas o aplicaciones que se deben utilizar para apoyar el aprendizaje. Los futuros docentes necesitan aprender de forma práctica el uso de las TIC y ampliar estos conocimientos a lo largo de todo su periodo formativo (Marín y Romero, 2009), incluyendo tanto experiencia práctica como desarrollo profesional (Silva, 2012). En este caso, conviene señalar que el uso de entornos personales de aprendizaje (PLE), redes sociales o plataformas e-learning, entre otros, pueden constituir recursos a tener en cuenta dentro de metodologías que favorezcan el aprendizaje y la integración tecnológica en el aula (Fernández y Barreira, 2017; Palomares y Garrote, 2010). Por último, no se pueden olvidar la multitud de herramientas y software disponibles para la creación de recursos didácticos y cuyo uso otorga un gran potencial para el personal docente. Estos recursos están incrementando notablemente su presencia en los contextos educativos y las publicaciones 
especializadas en estas temáticas (Quirós, 2009; Tucker, 2012). Como ejemplos representativos de este tipo de software se pueden mencionar: Filmora, Audacity, Utellstory o Plotagón, entre otros.

Si bien los beneficios de la utilización de estas aplicaciones están ampliamente contrastados, ya que gracias a estos recursos el deseo por aprender del alumnado se ve incrementado puesto que estimulan el aprendizaje colaborativo y propician la creatividad y el desarrollo del pensamiento crítico (Quirós, 2009; Segovia y Pavón, 2017), el éxito de su implantación depende en gran medida de la aceptación de las mismas entre el profesorado de los centros educativos (Sandoval, Rodríguez y Maldonado 2017). Y es esta aceptación pedagógica la que se debe trabajar desde la formación inicial docente, fomentando la familiarización de los futuros profesores con las distintas herramientas e impartiendo la capacitación necesaria para su correcta utilización. Además, es de necesidad obligada el estudio de la percepción y valoración del alumnado universitario en relación al uso e integración de las TIC en su proceso educativo (García-Valcárcel y Tejedor Tejedor, 2017; Morales, Ortiz, Trujillo y Raso, 2015), así como conocer las expectativas de los futuros docentes respecto a la inclusión de los diferentes recursos digitales en su formación y práctica profesional.

El estudio que se presenta a continuación pretende recoger las opiniones de los futuros docentes, en su formación inicial, respecto al aprendizaje de la aplicación Plotagón para diseñar y producir recursos didácticos audiovisuales que contemplen los diferentes elementos curriculares de la etapa de educación infantil. Este objetivo principal, se concreta en los siguientes objetivos específicos:

1. Describir las opiniones del alumnado de segundo curso del Grado de Educación Infantil acerca del aprendizaje del programa Plotagón para el diseño y producción de recursos didácticos audiovisuales.

2. Analizar si existen diferencias entre mujeres y hombres, así como entre los grupos de edad y los dispositivos utilizados por el alumnado para la visualización de contenidos audiovisuales respecto a la valoración que hacen de la experiencia.

3. Observar la existencia o no de correlaciones entre las diferentes dimensiones del cuestionario: Percepción personal sobre el recurso; Aportaciones del recurso al aprendizaje; Valoración del software
Plotagón para la elaboración del recurso audiovisual.

4. Establecer modelos para predecir la valoración del software Plotagón en función del aprendizaje vivido a través de regresiones lineales múltiples.

\section{MATERIAL Y MÉTODO}

\subsection{Muestreo y participantes}

La selección de la muestra se realizó utilizando el procedimiento de muestreo no probabilístico o de conveniencia (Cuenca y Lozano, 2016), debido a que la metodología de aprendizaje desarrollada en el aula, así como la aplicación del cuestionario, solo se pudo realizar a los grupos de estudiantes a los que los docentes de este estudio impartían clase durante el curso académico 2016-2017.

La muestra estuvo compuesta por 100 estudiantes de $2^{\circ}$ curso de Grado de Educación Infantil. En cuanto a la relación entre la edad y el sexo del alumnado, la mitad de los discentes (50\%) tenían entre $18-20$ años, siendo un $96 \%$ chicas y un $4 \%$ chicos. El 33\% tenían entre 21-23 años, del cual el $81.8 \%$ eran mujeres y el $12,2 \%$ hombres. Por otra parte, un 7\% tenían entre 24-26 años, del cual el $100 \%$ eran chicos. Finalmente, un $10 \%$ poseía más de 26 años, siendo, como el caso anterior, el 100\% chicas. En cuanto al dispositivo utilizado por el alumnado para la visualización de contenidos audiovisuales, destacar que $44 \%$ de los encuestados utilizaban principalmente el PC/MAC, el 24\% empleaban varios dispositivos, el $18 \%$ usaban el smartphone y un $14 \%$ la tableta digital.

\subsection{Instrumento de recogida de datos}

El instrumento utilizado para llevar a cabo este estudio, denominado Cuestionario sobre el aprendizaje del software Plotagón en educación superior, está compuesto por 27 ítems.

A través de los 27 ítems, medidos en escala Likert de 5 puntos (1=totalmente en desacuerdo a $5=$ totalmente de acuerdo), se preguntó a los estudiantes sobre su opinión acerca del aprendizaje del software Plotagón para la elaboración del recurso didáctico audiovisual. Además, se solicitó al alumnado que respondiese a una serie de preguntas directas que hacían alusión a sus datos personales (sexo y edad), así como de relación con los dispositivos utilizados cotidianamente para la visualización de contenido audiovisual.

La validación y fiabilidad del mismo han sido medidas y corroboradas mediante diferentes análisis 
estadísticos. En cuanto a la validez de constructo, se ha realizado un análisis factorial exploratorio, utilizando para la ejecución de esta prueba el método de Correlaciones Policóricas (Choi, Kim, Chen, y Dannels, 2011), junto con un proceso de extracción de factores comunes de "Mínimos Cuadrados No Ponderados" (ULS), considerando un procedimiento de rotación "Oblimin Ponderado" (Lorenzo-Seva, 2000), cuyo índice Kaiser-Meyer-Olkin (KMO) fue de ,91 y test de esfericidad de Barllett con $\mathrm{p}=, 000$. Por otra parte, la varianza presenta una distribución no normal, medida a través de la prueba de Kolmogorov-Smirnov $(p=, 004)$, El resultado de la extracción de los componentes principales refleja la existencia de tres factores, donde la varianza total explicada es de $63,5 \%$, lo cual revela un equilibrio apropiado entre los componentes del instrumento, representativos del concepto teórico. La medición de la fiabilidad del instrumento se ha llevado a cabo a través del enfoque de consistencia interna (MerinoSoto, 2016), obteniendo un Alfa de Cronbach de ,93, lo cual refleja una fiabilidad alta de los ítems expresados en el cuestionario. Por otro lado, la matriz de componentes rotados expresa que existen tres factores que tratan de aportar una explicación al constructo estudiado: 1. Percepción personal sobre el recurso. $(\alpha=, 90) ; 2$. Aportaciones del recurso al aprendizaje $(\alpha=, 92)$; 3. Valoración del software Plotagón para la elaboración del recurso $(\alpha=, 70)$.

Tabla 1. Matriz de componentes rotados en el análisis factorial exploratorio.

\begin{tabular}{l|lll} 
VAR & $\begin{array}{l}\text { Dimensión } \\
\text { Percepción } \\
\text { personal }\end{array}$ & $\begin{array}{l}\text { Dimensión 2: } \\
\text { Aportaciones } \\
\text { aprendizaje }\end{array}$ & $\begin{array}{l}\text { Dimensión } \\
\text { Valoración } \\
\text { software }\end{array}$ \\
\hline $\mathbf{1}$ &, 705 & & \\
$\mathbf{2}$ &, 922 & & \\
$\mathbf{3}$ &, 774 & & \\
$\mathbf{4}$ &, 650 & & \\
$\mathbf{5}$ &, 654 & & \\
$\mathbf{6}$ &, 509 & & \\
$\mathbf{7}$ &, 586 & & \\
$\mathbf{8}$ &, 670 & & \\
$\mathbf{9}$ &, 387 &, 320 & \\
$\mathbf{1 0}$ & &, 416 & \\
$\mathbf{1 1}$ & &, 539 & \\
$\mathbf{1 2}$ & &, 507 & \\
$\mathbf{1 3}$ & &, 614 & \\
$\mathbf{1 4}$ & &, 304 & \\
$\mathbf{1 5}$ & &, 408 & \\
$\mathbf{1 6}$ & &, 420 & \\
$\mathbf{1 7}$ & &, 444 & \\
$\mathbf{1 8}$ & &, 337 & \\
$\mathbf{1 9}$ & &, 390 & \\
$\mathbf{2 0}$ & &, 391 & \\
$\mathbf{2 1}$ & & & \\
$\mathbf{2 2}$ & & &
\end{tabular}

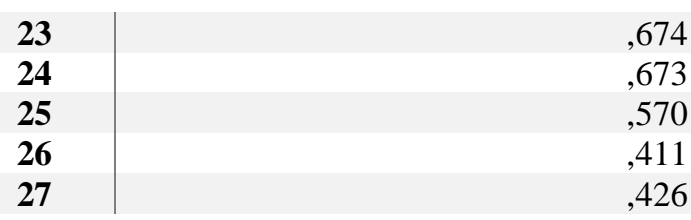

\subsection{Procedimiento}

El instrumento fue facilitado a los estudiantes al concluir la dinámica de aprendizaje basada en el programa Plotagón para la elaboración de recursos didácticos audiovisuales, dedicando 30 minutos para su cumplimentación.

\subsection{Análisis de datos}

Los análisis que hemos llevado a cabo en este estudio y mediante los cuales hemos pretendido dar respuesta a los objetivos planteados son los siguientes:

- En primer lugar, se ha realizado un análisis descriptivo de las 27 variables que componían el cuestionario a través de los estadísticos de medida central (media) y de medida de dispersión (desviación típica).

- En segundo lugar, se ha llevado a cabo diferentes análisis de varianza para comprobar si existía relación entre las dimensiones del cuestionario y las variables independientes: sexo, edad y dispositivo de visualización de contenidos audiovisuales. Para ello, se llevaron a cabo U de Mann Whitney y K de Kuskal Wallis, utilizando el paquete estadístico SPSS 23.

- En tercer lugar, se ha procedido a comprobar la relación entre las dimensiones que componían el cuestionario mediante correlaciones bivariadas.

- Por último, se han desarrollado regresiones lineales múltiples para predecir la valoración del software Plotagón para la elaboración de recursos didácticos en función del aprendizaje vivido.

\section{RESULTADOS}

En primer lugar, presentamos los resultados descriptivos de los 27 ítems que componen el cuestionario, utilizado en el presente trabajo de investigación. En la tabla 2 podemos observar las medias y desviaciones típicas de los ítems que componen el cuestionario sobre el uso del software Plotagón en la elaboración de recursos didácticos audiovisuales en educación superior. 
Tabla 2. Distribución de frecuencias de los ítems del cuestionario

\begin{tabular}{|c|c|c|c|}
\hline $\mathrm{D}$ & Ítem & M & DT \\
\hline \multirow{9}{*}{ 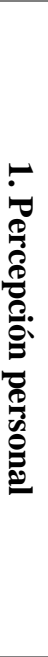 } & 1. Resulta un recurso divertido & 4,64 & ,594 \\
\hline & $\begin{array}{l}\text { 2. Puede mantener la atención del } \\
\text { alumnado }\end{array}$ & 4,67 &, 551 \\
\hline & $\begin{array}{l}\text { 3. Facilita la comprensión de hechos } \\
\text { y sentimientos }\end{array}$ & 4,28 & ,792 \\
\hline & $\begin{array}{l}\text { 4. Fomenta la creatividad de niños y } \\
\text { niñas }\end{array}$ & 4,54 & ,642 \\
\hline & 5. Potencia la iniciativa del alumnado & 4,40 & ,681 \\
\hline & $\begin{array}{l}\text { 6. Desarrolla hábitos de sensibilidad } \\
\text { artística }\end{array}$ & 4,20 & ,791 \\
\hline & $\begin{array}{l}\text { 7. Constituye un recurso motivador } \\
\text { para el alumnado }\end{array}$ & 4,64 &, 559 \\
\hline & $\begin{array}{l}\text { 8. Fomenta el entretenimiento y la } \\
\text { relajación del alumnado }\end{array}$ & 4,41 & ,697 \\
\hline & $\begin{array}{l}\text { 9. Potencia la transmisión de roles, } \\
\text { creencias y valores }\end{array}$ & 4,44 & ,729 \\
\hline \multirow{12}{*}{ 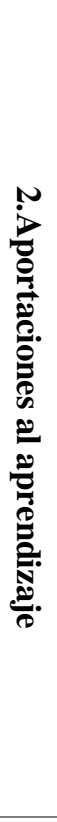 } & 10. Desarrolla el lenguaje oral & 4,42 & ,669 \\
\hline & 11. Desarrolla el lenguaje escrito & 3,47 & ,999 \\
\hline & 12. Permite ampliar el léxico & 4,09 &, 865 \\
\hline & 13. Desarrolla la afectividad & 4,19 & ,677 \\
\hline & $\begin{array}{l}\text { 14. Fomenta el proceso de } \\
\text { socialización }\end{array}$ & 4,38 & ,693 \\
\hline & $\begin{array}{l}\text { 15. Permite simular situaciones de la } \\
\text { vida cotidiana }\end{array}$ & 4,64 &, 594 \\
\hline & $\begin{array}{l}\text { 16. Fomenta la capacidad de toma de } \\
\text { decisiones }\end{array}$ & 4,26 & ,719 \\
\hline & $\begin{array}{l}\text { 17. Desarrolla los procesos de } \\
\text { atención, diálogo y escucha }\end{array}$ & 4,54 & ,642 \\
\hline & $\begin{array}{l}\text { 18. Permite desarrollar los objetivos } \\
\text { que establece el curriculum oficial }\end{array}$ & 4,34 & ,713 \\
\hline & $\begin{array}{l}\text { 19. Permite desarrollar los contenidos } \\
\text { que establece el curriculum oficial }\end{array}$ & 4,31 & ,706 \\
\hline & $\begin{array}{l}\text { 20. Facilita la adquisición de } \\
\text { habilidades/destrezas para } \\
\text { la resolución de problemas }\end{array}$ & 4,27 & ,694 \\
\hline & 21. Fomenta la autonomía personal & 4,12 &, 844 \\
\hline \multirow{6}{*}{ 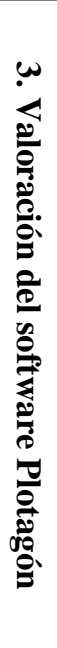 } & $\begin{array}{l}\text { 22. Plotagón facilita la elaboración } \\
\text { del recurso audiovisual. }\end{array}$ & 4,54 &, 757 \\
\hline & $\begin{array}{l}\text { 23. Plotagón reduce el tiempo } \\
\text { empleado en la } \\
\text { elaboración del recurso audiovisual. }\end{array}$ & 4,23 & ,972 \\
\hline & $\begin{array}{l}\text { 24. Plotagón tiene un entorno } \\
\text { intuitivo y fácil de usar }\end{array}$ & 4,08 & ,960 \\
\hline & $\begin{array}{l}\text { 25. El aprendizaje de la aplicación } \\
\text { me ha llevado tiempo }\end{array}$ & 2,74 & 1,21 \\
\hline & $\begin{array}{l}\text { 26. Plotagón permite llevar a cabo } \\
\text { dinámicas de aprendizaje } \\
\text { cooperativo }\end{array}$ & 4,43 & 670 \\
\hline & $\begin{array}{l}\text { 27. He tenido dificultades en la } \\
\text { elaboración del recurso } \\
\text { Audiovisual a través de la aplicación } \\
\text { Plotagón }\end{array}$ & 2,95 & 1,29 \\
\hline
\end{tabular}

A continuación, presentamos los resultados agrupados en función de las 3 dimensiones de las que consta el cuestionario. En este sentido, mostramos los datos descriptivos de cada una de las dimensiones, así como la existencia de diferencias estadísticamente significativas, atendiendo a las variables independientes del estudio (sexo, edad y dispositivo empleado para la visualización de contenidos). Finalmente, se presenta la relación existente entre las dimensiones que componen el cuestionario, así como el establecimiento de un modelo predictor de la valoración del software Plotagón respecto a la dinámica de aprendizaje vivida por el alumnado.

\subsection{Percepción personal del alumnado sobre el recurso}

En esta dimensión, agrupamos 9 ítems que hacían alusión a las percepciones del alumnado en cuanto a las posibilidades del recurso audiovisual, como pueden ser la diversión, atención, motivación, relajación, creatividad, iniciativa, comprensión de hechos y sentimientos, etc.

En los resultados obtenidos en esta dimensión, se observó que el alumnado manifiesta una opinión parcialmente de acuerdo en cuanto a su percepción respecto a las posibilidades del recurso audiovisual $(\mu=4,46 ; \sigma=, 509)$.

En cuanto a las diferencias que el sexo establecía en relación a las percepciones del alumnado respecto a las posibilidades del recurso audiovisual, la prueba U de Mann Whitney para muestras independientes, señaló que existían diferencias estadísticamente significativas en función del sexo de los estudiantes $[U=161,000,(8,92), \mathrm{z}=-2,249, \mathrm{p}<, 01] . \quad$ En este sentido, las chicas presentaban unos rangos promedios superiores a los de los chicos en relación a las percepciones personales.

Por otra parte, para determinar la influencia de la edad, así como el dispositivo usado para la visualización de contenidos audiovisuales, sobre los resultados, se han realizado comparaciones múltiples de media a través de la prueba $\mathrm{K}$ de Kruskal Wallis. Los resultados obtenidos mostraron que no había diferencias estadísticamente significativas entre la edad $\mathrm{y}$ las percepciones personales sobre las posibilidades de los recursos audiovisuales.

En cuanto a la relación del dispositivo empleado para la visualización de contenidos digitales y las percepciones personales del alumnado, los análisis mostraron que tampoco existían diferencias estadísticamente significativas. 


\subsection{Aportaciones del recurso al aprendizaje}

Esta dimensión está compuesta por 12 ítems en los que se valora las aportaciones de los recursos elaborados por el software Plotagón en el ámbito del aprendizaje. Concretamente, se alude a la comprensión oral y escrita, enriquecimiento léxico, mejora en la resolución de problemas, consecución de los diferentes elementos del diseño curricular, etc.

En los resultados obtenidos en esta dimensión, observamos que el alumnado manifiesta una valoración de parcialmente de acuerdo respecto a la dimensión "Aportaciones del recurso al aprendizaje $(\mu=4,62 ; \sigma=, 58)$.

Por otra parte, la prueba U de Mann Whitney realizada para identificar posibles diferencias en cuanto al sexo del alumnado en cuanto a la valoración del recurso en el ámbito del aprendizaje, indicó que existían diferencias estadísticamente significativas $[U=159,000,(8,92), \mathrm{z}=-2,660, \mathrm{p}<, 01]$. En este sentido, las chicas presentaban unos rangos promedios superiores a los de los chicos en relación a las aportaciones del recurso al aprendizaje.

Por otra parte, los análisis de varianza ( $\mathrm{K}$ de Kruskal Wallis) realizados para constatar la existencia de diferencias significativas entre la edad y el dispositivo usado para la visualización de contenidos audiovisuales sobre los resultados en relación a las aportaciones del recurso en el ámbito del aprendizaje, mostraron que no existían diferencias estadísticamente significativas en relación a la edad. En cuanto a la influencia del dispositivo empleado por el alumnado para la visualización de contenidos audiovisuales, respecto a las aportaciones del recurso en el aprendizaje, los análisis tampoco arrojaron diferencias significativas.

\subsection{Valoración del software Plotagón en la elaboración de recursos didácticos audiovisuales}

Esta dimensión está compuesta por 6 ítems en los que se valora el programa Plotagón empleado para la realización y producción de contenidos didácticos digitales. Específicamente, se hace referencia a aspectos como el entorno de la aplicación, dificultades surgidas durante el dominio de la aplicación, tiempo empleado o facilidad para crear dinámicas de aprendizaje cooperativo, entre otros.

En los resultados obtenidos en esta dimensión, observamos que el alumnado manifiesta una valoración de indiferencia respecto a la dimensión "Valoración del Software Plotagón en la elaboración de recursos didácticos audiovisuales" $(\mu=3,82 ; \sigma=$ $0,64)$.
La prueba U de Mann Whitney realizada para identificar posibles diferencias en relación al sexo del alumnado en cuanto a la valoración de Plotagón en la elaboración de recursos digitales, mostró que no existían diferencias estadísticamente significativas.

Por otra parte, los análisis de varianza ( $\mathrm{K}$ de Kruskal Wallis) realizados para constatar la existencia de diferencias significativas entre la edad y el dispositivo utilizado para visualizar contenidos multimedia en relación a la valoración del software Plotagón, mostraron que no existían diferencias estadísticamente significativas en ningún caso.

\subsection{Análisis Correlacional}

En este apartado, abordaremos el estudio correlacional entre las 3 dimensiones del cuestionario. Los datos resultantes de la aplicación de la prueba de correlación de Pearson, realizada para comprobar la relación entre las 3 dimensiones de la escala pueden verse en la tabla 3.

Tabla 3. Resultados de las correlaciones bivariadas de las dimensiones del cuestionario sobre el aprendizaje del software Plotagón en educación superior

\section{Correlaciones}

\begin{tabular}{|c|c|c|c|c|}
\hline & & Dim. 1 & Dim. 2 & Dim. 3 \\
\hline \multirow{3}{*}{$\begin{array}{l}\text { Dimensión 1: } \\
\text { Percepción } \\
\text { personal del } \\
\text { alumnado }\end{array}$} & $\begin{array}{l}\text { Correlación } \\
\text { de Pearson }\end{array}$ & 1 &, $836^{* *}$ &, $444^{* *}$ \\
\hline & $\begin{array}{l}\text { Sig. } \\
\text { (bilateral) }\end{array}$ & &, 000 & ,000 \\
\hline & $\mathrm{N}$ & 100 & 100 & 100 \\
\hline \multirow{3}{*}{$\begin{array}{l}\text { Dimensión 2: } \\
\text { Aportaciones } \\
\text { Aprendizaje }\end{array}$} & $\begin{array}{l}\text { Correlación } \\
\text { de Pearson }\end{array}$ &, $836^{* *}$ & 1 &, $406^{* *}$ \\
\hline & $\begin{array}{l}\text { Sig. } \\
\text { (bilateral) }\end{array}$ & ,000 & & ,000 \\
\hline & $\mathrm{N}$ & 100 & 100 & 100 \\
\hline \multirow{3}{*}{$\begin{array}{l}\text { Dimensión 3: } \\
\text { Valoración } \\
\text { Plotagón }\end{array}$} & $\begin{array}{l}\text { Correlación } \\
\text { de Pearson }\end{array}$ &, $444^{* *}$ &, $406^{* *}$ & 1 \\
\hline & $\begin{array}{l}\text { Sig. } \\
\text { (bilateral) }\end{array}$ &, 000 & ,000 & \\
\hline & $\mathrm{N}$ & 100 & 100 & 100 \\
\hline
\end{tabular}

**. La correlación es significativa en el nivel ,01 (bilateral).

En función de los datos obtenidos, podemos afirmar que existe relación entre la dimensión 1 (Percepción personal del alumnado respecto a los recursos audiovisuales generados a través de Plotagón) con la dimensión 2 (Aportaciones de los recursos digitales al ámbito del aprendizaje) y la 3 (Valoración del software Plotagón en la elaboración del recurso audiovisual) respectivamente $(\mathrm{R}=, 836 \mathrm{y}$ $\mathrm{p}=, 000 ; \mathrm{R}=, 444$ y $\mathrm{p}=, 000$ ), dado el nivel de significatividad bilateral de n.s=,01. La relación 
entre las mismas es alta, en el primer caso y moderada en el segundo, tal y como señalan Mateo (2004) y Pérez, García, Gil y Galán (2009).

Por otra parte, podemos afirmar que, además, existe relación entre la dimensión 2 (Aportaciones de los recursos digitales al ámbito del aprendizaje) con la dimensión 3 (Valoración del software Plotagón en la elaboración del recurso audiovisual) $(\mathrm{R}=, 406$ y $\mathrm{p}=, 000$ ), dado que hay nivel de significatividad bilateral al n.s=,01. La relación entre las mismas es moderada, como señalan los autores mencionados anteriormente.

\subsection{Modelos explicativos de la valoración del software Plotagón en el aprendizaje.}

En este punto, tratamos de explicar la variable "Valoración del software Plotagón en la elaboración del recurso audiovisual" en función de las medidas de la dimensión "Percepción personal del alumnado respecto a los recursos audiovisuales generados a través de Plotagón" y la dimensión "Aportaciones de los recursos digitales al ámbito del aprendizaje" a través de regresiones lineales múltiples (Pardo y Ruiz, 2002), utilizando el método "por pasos", con el fin de observar las variables predictoras y su relación con la variable criterio.

Tabla 4. Coeficientes de la recta de regresión para la variable dependiente "Valoración del software Plotagón en la elaboración del recurso audiovisual" en función de

las medidas de la dimensión "Percepción personal del alumnado respecto a los recursos audiovisuales generados a través de Plotagón".

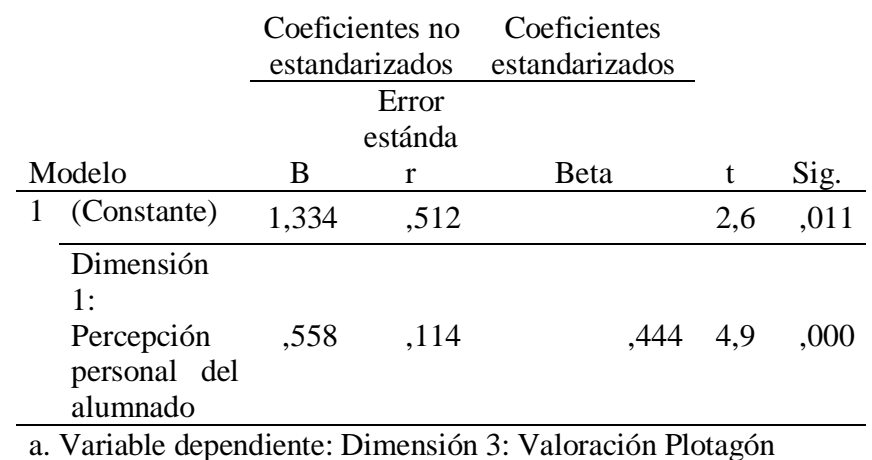

Los resultados obtenidos, tal y como podemos ver en la tabla 4, nos permiten observar que la dimensión "Percepción personal del alumnado respecto a los recursos audiovisuales generados a través de Plotagón" constituye un factor predictor de la dimensión "Valoración del software Plotagón en la elaboración del recurso audiovisual, ya que $\beta=, 444, \quad \mathrm{t}(444)=4,90, \quad \mathrm{p}<, 01 \quad \mathrm{y}$, por tanto, es estadísticamente significativa, por lo que aceptamos la hipótesis de relación lineal entre la percepción personal del alumnado respecto a los recursos audiovisuales y la valoración del software Plotagón en la elaboración del recurso audiovisual. Por último, indicar que el valor de $\mathrm{R}^{2}$ fue de, 189 , por lo que el $18,9 \%$ de la variabilidad de la valoración de dicho software es explicada por la percepción personal del alumnado respecto a los recursos audiovisuales.

\section{DISCUSIÓN}

El análisis descriptivo de las percepciones de los estudiantes acerca del aprendizaje del programa Plotagón, para el diseño de recursos audiovisuales, indicó que el alumnado percibe que el recurso es divertido, motivador, relajante y, además, señala que potencia la creatividad, la iniciativa personal y la comprensión de hechos y sentimientos. En cuanto a las aportaciones de los recursos elaborados por el software Plotagón en el ámbito del aprendizaje, los estudiantes señalaron que mejora la comprensión oral y escrita, enriquece el léxico, mejora la resolución de problemas y ayuda en la consecución de los diferentes elementos del diseño curricular, entre otros aspectos. En lo referente a la valoración del software en la elaboración de recursos didácticos audiovisuales, el alumnado señaló que la elaboración de recursos no les planteaba dificultades, que no les llevaba demasiado tiempo y que le facilitaba la creación de dinámicas de aprendizaje cooperativo, entre otros. En este sentido, los datos concuerdan con estudios anteriores (Dans y Muñoz, 2016; Meter, 2004), en los que se señala la importancia de la percepción y valoración que los futuros docentes tengan de los recursos TIC y de las aportaciones de los mismos en su práctica profesional.

Seguidamente, se comprobó si existían diferencias en las dimensiones del cuestionario en relación con el sexo, la edad y los dispositivos utilizados para la visualización de contenido audiovisual. En este caso, los resultados han apuntado que el primer caso si imprimía diferencias significativas en dos de las tres dimensiones del cuestionario. En concreto, las chicas manifestaron en mayor proporción que los chicos una percepción personal más positiva del recurso y una valoración más positiva de las aportaciones del mismo a las prácticas educativas; sin embargo, no se encontraron diferencias entre unos y otras en cuanto a la valoración que hacían del software Plotagón en la elaboración de recursos didácticos digitales. Estos datos no se han podido contrastar con estudios anteriores, ya que no se han encontrado 
investigaciones que hayan analizado las diferencias que el sexo establece en cuanto a las percepciones que tienen los futuros docentes del uso de la aplicación Plotagón. Por su parte, la edad y los dispositivos utilizados no señalaron diferencias en ninguna de las dimensiones estudiadas.

En cuanto a la relación de las dimensiones del cuestionario, existe correlación en todas ellas. No obstante, la más alta se encontró entre la percepción personal sobre el recurso y las aportaciones que el recurso tiene al aprendizaje. Esto tiene sentido, según los datos de investigaciones previas, donde se señala la relación entre una percepción positiva hacia las TIC y las aportaciones que las mismas tengan al aprendizaje (Fernández y Barreira, 2017; Palomares y Garrote, 2010; Roblizo y Cózar, 2015).

Finalmente, el análisis de regresiones indicó que la percepción personal del alumnado respecto a los recursos audiovisuales generados a través de Plotagón constituye un factor predictor de la dimensión "Valoración del software Plotagón en la elaboración del recurso audiovisual". Estos datos tampoco han podido contrastarse con estudios anteriores, ya que este tipo de análisis se están llevando a cabo recientemente dentro de los estudios pertenecientes al ámbito de las Ciencias de la Educación.

\section{CONCLUSIONES}

La literatura científica apunta que la incorporación adecuada de las TIC a la educación afronta todavía importantes retos (Blanco y Anta, 2016) ya que, si bien es cierto que los centros educativos cuentan cada vez con más recursos digitales (Castells, 2002; Perrenoud, 2004), en algunos casos la utilización de los mismos no es del todo acertada, puesto que se realizan prácticas rutinarias donde se le imprime más importancia a los aspectos técnicos que a los pedagógicos. En este sentido, conviene aclarar que no es suficiente con tener recursos tecnológicos y acceso a los mismos por parte del profesorado y del alumnado, sino que el uso que se haga de los mismos debe ser efectivo y pedagógico (Marín y Romero, 2009), es decir, que las TIC se incorporen con naturalidad en las prácticas educativas dentro y fuera del aula. Por tanto, resulta fundamental la formación de los docentes (Cabero, 2010), ya que serán estos los que incorporen con menor o mayor éxito las mismas en su quehacer diario en las aulas y con sus estudiantes. Consecuentemente, la efectividad y eficacia de los procesos de enseñanzaaprendizaje del alumnado va a depender de la formación y capacidades con las que cuente el profesorado que les enseñe a interactuar de manera natural con los recursos digitales en las dinámicas de aprendizaje (Maldonado, 2012). De ahí, la importancia de que tanto la formación inicial docente como la permanente, contemplen dentro de sus programas de capacitación diferentes aspectos que permitan a los profesionales de la educación el desarrollo de las capacidades, habilidades y destrezas necesarias para la integración de dichos recursos en dinámicas de aprendizaje de cualquier etapa educativa.

En este sentido, el docente se entiende como una entidad facilitadora del proceso de aprendizaje que tiene que afrontar los retos tecnológicos que marca la sociedad actual, sumado a la constante aparición de nuevos recursos digitales, así como la complejidad de los mismos (Quirós, 2009; Tucker, 2012). Por tanto, el profesorado no solo debe contar con la formación inicial que debe incluirse en los planes de estudio, sino que la capacitación ha de ser permanente para poder responder así a las demandas tecnológicas de la sociedad actual del conocimiento.

Todos estos cambios implican una transformación en los procesos educativos, manifestándose nuevas opciones para hacer más eficaces los procesos de enseñanza aprendizaje. $\mathrm{Al}$ respecto, existen un gran número de recursos para dar respuesta educativa a la revolución tecnológica que estamos experimentando y suponen un reto para los educadores que deben aprender y enseñar el uso de los mismos para hacer más fácil y dinámico el proceso educativo (Sandoval, Rodríguez y Maldonado 2017).

En definitiva, es importante que los futuros docentes sean conscientes de la importancia que estos recursos didácticos digitales tienen en el proceso de enseñanza y aprendizaje, ya que suponen una magnifica innovación pedagógica que conllevará a aprendizajes más significativos en su alumnado (Morales, Ortiz, Trujillo y Raso, 2015). Una mayor concienciación, así como una formación inicial y permanente del profesorado en TIC puede reflejarse en una valoración o percepción más positiva de las aportaciones al aprendizaje de los recursos audiovisuales.

\section{REFERENCIAS}

Adell, J. (2004). Internet en el aula. Edutec: Revista Electrónica de Tecnología Educativa, 17, 1-26. Recuperado de http://www.edutec.es/revista/index.php/edutece/article/view/530/264

Blanco, A., y Anta, P. (2016). La perspectiva de estudiantes en línea sobre los entornos virtuales de aprendizaje en la educación superior. Innoeduca. International Journal of 
Technology and Educational Innovation, 2(2), 109-116, doi:10.20548/innoeduca.2016.v2i2.1062

Boza, A. y Conde, S. (2015). Web 2.0 en educación superior: formación, actitud, uso, impacto, dificultades y herramientas. Digital Education Review, 28, 45-58. Recuperado de http://greav.ub.edu/der

Cabero, J. (2010). Retos de la integración de las TICs en los procesos educativos. Límites y posibilidades. Perspectiva Educacional, 49(1), 32-61.

Cabero, J. y Marín, V. (2012). ICT training of university teachers in a Personal Learning Environment. Project DIPRO 2.0. NAER, 1(1), 2-6, doi:10.7821/naer.1.1.2-6

Castells, M. (2002). La era de la información. La sociedad red (Vol. I). México, D.F: Siglo XXI.

Choi, J., Kim, S., Chen, J., y Dannels, S. (2011). A comparison of maximum likelihood and Bayesian estimation for polychoric correlation using Monte Carlo simulation. Journal of Educational and Behavioral Statistics, 36, 523-549. doi:10.3102/1076998610381398

Cuenca, A. y Lozano, S. (2016). La enseñanza de la investigación. Diálogo entre la teoría y el oficio del investigador en Trabajo Social. La Plata: Editorial de la Universidad Nacional de La Plata (EDULP)

Dans, I., y Muñoz, P.C. (2016). Las redes sociales como motivación para el aprendizaje: opinión de los adolescentes. Innoeduca. International Journal of Technology and Educational Innovation, 2(1), 20-28.

Escudero, J.M., Martínez-Domínguez, B., Nieto, J.M. (2018). Las TIC en la formación continua del profesorado en el contexto español. Revista de Educación, 382, 57-80, doi:10.4438/1988-592X-RE-2018-382-392

EURYDICE (2011). Cifras clave sobre el uso de las TIC para el aprendizaje y la innovación en los centros escolares de Europa 2011. Madrid: Ministerio de Educación.

Fernández, A., y Barreira, A. (2017). El cortometraje como herramienta innovadora para el alumnado con Altas Capacidades en Educación Primaria.Innoeduca. International Journal of Technology and Educational Innovation, 3(1), 28-36, doi:10.24310/innoeduca.2017.v3i1.1962

García-Valcárcel, A. y Tejedor Tejedor, F. J. (2017). Percepción de los estudiantes sobre el valor de las TIC en sus estrategias de aprendizaje y su relación con el rendimiento. Educación XX1, 20(2), 137-159, doi:10.5944/educXX1.19035

González, R., García, F. y Gonzalo, N. (2011). Los edublogs como herramienta facilitadora en comunidades virtuales de aprendizaje. Relada. Revista Electrónica de ADA, 5(3), 248-256.

Lorenzo-Seva, U. (2000). The weighted oblimin rotation. Psychometrika, 65, 301-318.

Maldonado, G. A. (2012). Actitudes con respecto al uso de la plataforma tecnológica de teleformación Moodle: El caso de los estudiantes de la Facultad de Ciencias de la Educación.

Recuperado http://helvia.uco.es/xmlui/handle/10396/7194

Marín, V. (2014). Aprendiendo a través de los videojuegos. La opinión de los y las jóvenes educadores y educadoras. Revista de Estudios de Juventud, 106, 165-149.

Marín, V., y Romero, M. A. (2009). La formación docente universitaria a través de las TICs. Pixel-Bit. Revista de Medios y Educación, 35, 97-103.

Marín, V., y Sampedro, B. E. (2016). Innovando en el aula de Educación Primaria con GT 6. Innoeduca. International Journal of Technology and Educational Innovation, 2(1), 13-19, doi:10.20548/innoeduca.2016.v2i1.1061
Mateo, J. (2004). La investigación 'ex post-facto'. En R. Bisquerra, (coord.), Metodología de investigación educativa (pp. 196-230). Madrid: La Muralla,

Merino-Soto, C. (2016). Differences between Cronbach's alpha coefficients, with small samples and parts: a VB program. Anales de Psicología, 32(2), 587-588.

Meter, D. (2004). Desarrollo social y educativo con las nuevas tecnologías. En F. Martínez y M. Prendes (Coord.), Nuevas tecnologías y Educación (pp. 69-84). Madrid: Pearson.

Morales, M., Ortiz, A. M., Trujillo, J. M., y Raso, F. (2015). Percepción del alumnado universitario acerca del uso e integración de las TIC en el proceso educativo de la Facultad de Educación de Granada. Innoeduca. International Journal of Technology and Educational Innovation, 1(2), 57-68.

Palomares, A. y Garrote. D. (2010). Competencias básicas y nuevo modelo de evaluación. En M. Marín (coord.), Evaluación de competencias en los nuevos grados (pp.147-151). Ciudad-Real: Universidad de Castilla-La Mancha.

Pardo, A., y Ruiz, M. (2002). SPSS 11: Guía para el análisis de datos. Madrid: McGraw-Hill.

Pérez, R., García, J.L., Gil, J.A., y Galán, A. (2009). Estadística aplicada a la Educación. Madrid: UNED - Pearson.

Perrenoud, P. (2004). Diez nuevas competencias para enseñar. Barcelona: Grao.

Quirós, E. (2009). Recursos didácticos digitales: medios innovadores para el trabajo colaborativo en línea. Revista Electrónica Educare, 13(2), 47-62.

Roblizo, M. y Cózar, R. (2015). Usos y competencias en TIC en los futuros maestros de Educación Infantil y Primaria: hacia una alfabetización tecnológica real para docentes. Pixel Bit. Revista de Medios y Educación, 47, 23-39, doi:10.12795/pixelbit.2015.i47.02

Sancho, J., y Hernández, F. (2006). Tecnologias para transformar a educação.Porto Alegre: Artmed Editora S.A

Sandoval, P., Rodríguez, F., y Maldonado, A. (2017). Evaluación de la alfabetización digital y pedagógica en TIC, a partir de las opiniones de estudiantes en Formación Inicial Docente. ducação e Pesquisa,43(1), 127-143.

Santiago, R., Navaridas, F. y Repáraz, R. (2014). La escuela 2.0: La percepción del docente en torno a su eficacia en los centros educativos de La Rioja. Educación XX1, 17(1), 243-270, doi:10.5944/educxx1.17.1.10713

Segovia, B. y Pavón, C. (2017). Creación audiovisual para comprender Europa en educación secundaria. Aportaciones desde un proyecto Erasmus+. EDMETIC, 6(2), 10-26, doi:10.21071/edmetic.v6i2.5790

Silva, J. (2012). Estándares TIC para la formación inicial docente: una política pública en el contexto chileno. Archivos Analíticos de Politicas Educativas, Arizona, 20(7), 1-35.

Tucker, B. (2012). The flipped classroom: Online instruction at home frees class time for learning. Education Next, 12(1). Recuperado de http://educationnext.org/theflipped-classroom/

Vásquez-Cuperio, S. y López-Penedo, S. (2016). Escuela, TIC e innovación educativa. Digital Education Review, 30, 248-261. Recuperado de http://revistes.ub.edu/index.php/der/article/view/15367/p df 